\section{Kidney Blood Pressure Research}

\title{
Role of Cytosolic Serine Hydroxymethyl Transferase 1 (SHMT1) in Phosphate- Induced Vascular Smooth Muscle Cell Calcification
}

\author{
Beate Boehme ${ }^{a}$ Nadeshda Schelski ${ }^{a}$ Manousos Makridakis ${ }^{b}$ Laura Henze $^{a}$ \\ Antonia Vlahou ${ }^{b}$ Florian Lang $^{c}$ Burkert Pieske ${ }^{a, d, e, f}$ Ioana Alesutan ${ }^{a, d, f}$ \\ Jakob Voelk|a,f \\ aDepartment of Internal Medicine and Cardiology, Charité - Universitätsmedizin Berlin, Campus \\ Virchow-Klinikum, Berlin, Germany, ${ }^{b}$ Biomedical Research Foundation, Academy of Athens, Athens, \\ Greece, 'Department of Physiology I, Eberhard-Karls University, Tübingen, 'Berlin Institute of Health \\ (BIH), Berlin, eDepartment of Internal Medicine and Cardiology, German Heart Institute Berlin, Berlin, \\ 'DZHK (German Centre for Cardiovascular Research), partner site Berlin, Berlin, Germany
}

\section{Key Words}

SHMT1 • Phosphate $\bullet$ Oxidative stress $\bullet$ Vascular calcification $•$ Osteo-/chondrogenic signaling - Vascular smooth muscle cells

\begin{abstract}
Background/Aims: Hyperphosphatemia promotes medial vascular calcification, at least partly, by induction of osteo-/chondrogenic transdifferentiation of vascular smooth muscle cells (VSMCs). The complex signaling pathways regulating this process are still incompletely understood. The present study investigated the role of cytosolic serine hydroxymethyl transferase 1 (SHMT1) in phosphate-induced vascular calcification. Methods: Endogenous expression of SHMT1 was suppressed by silencing in primary human aortic smooth muscle cells (HAoSMCs) followed by treatment without and with phosphate or antioxidants. Results: In HAoSMCs, SHMT1 mRNA expression was up-regulated by phosphate. Silencing of SHMT1 alone was sufficient to induce osteo-/chondrogenic transdifferentiation of HAoSMCs, as shown by increased tissue-nonspecific alkaline phosphatase (ALPL) activity and osteogenic markers MSX2, CBFA1 and ALPL mRNA expression. Furthermore, phosphate-induced ALPL mRNA expression and activity as well as calcification were augmented in SHMT1 silenced HAOSMCs as compared to negative control siRNA transfected HAOSMCs. Silencing of SHMT1 decreased total antioxidant capacity and up-regulated NADH/NADPH oxidase system components NOX4 and CYBA mRNA expression in HAoSMCs, effects paralleled by increased mRNA expression
\end{abstract}




\section{Kidney Blood Pressure Research}

Kidney Blood Press Res 2018;43:1212-1221

\begin{tabular}{l|l}
\hline DOI: $10.1159 / 000492248$ & (C) 2018 The Author(s). Published by S. Karger AG, Basel
\end{tabular}

Published online: 31 July, 2018 www.karger.com/kbr

Boehme et al.: SHMT1 and Vascular Calcification

of matrix metalloproteinase $M M P 2$ as well as $B A X / B C L 2$ ratio. More importantly, additional treatment with antioxidants TEMPOL or TIRON blunted the increased osteogenic markers mRNA expression in SHMT1 silenced HAoSMCs. Conclusion: Silencing of SHMT1 promotes osteo-/chondrogenic signaling in VSMCs, at least in part, by inducing cellular oxidative stress. It thus aggravates phosphate-induced calcification of VSMCs. The present findings support a regulatory role of SHMT1 in vascular calcification during conditions of hyperphosphatemia such as chronic kidney disease.

(C) 2018 The Author(s)

Published by S. Karger AG, Basel

\section{Introduction}

Medial vascular calcification is strongly associated with cardiovascular morbidity and mortality in chronic kidney disease (CKD) patients [1]. In these patients, impaired renal phosphate excretion leads to hyperphosphatemia, a major pathological factor promoting the initiation and progression of vascular calcification [2,3]. Accordingly, plasma phosphate concentrations are predictive for an increased risk for cardiovascular events and mortality $[4,5]$.

The pathological deposition of calcium-phosphate in the media of the arterial wall is an active process, with similarities to physiological bone mineralization [6, 7]. Vascular calcification is promoted mainly by vascular smooth muscle cells (VSMCs) [2, 8, 9]. Pathological factors, especially phosphate, trigger the transdifferentiation of VSMCs into an osteoblast and chondroblast-like phenotype $[2,6,10]$ including increased expression and activity of the osteogenic transcription factors msh homeobox 2 (MSX2) and core-binding factor $\alpha-1$ (CBFA1) [11,12] as well as osteogenic enzymes such as tissue-nonspecific alkaline phosphatase (ALPL) $[9,13]$ to initiate mineral deposition in the vascular tissue. The complex intracellular signaling pathways mediating osteo-/chondrogenic transdifferentiation of VSMCs are still incompletely understood.

The cytosolic serine hydroxymethyl transferase 1 (SHMT1) catalyzes the reversible conversion of serine and tetrahydrofolate to glycine and 5, 10-methylene tetrahydrofolate $[14,15]$ and is a critical enzyme in one carbon (methyl) metabolism [14-17]. One carbon transfer is a key component in amino acid and nucleotide synthesis as well as methylation of proteins, DNA and RNA $[18,19]$ and, thus, participating in the regulation of cell function, proliferation and growth $[18,20]$. SHMT1 has been associated with apoptotic pathways [21]. SHMT1 differs in targets and effects from the mitochondrial isoform SHMT2 [14, 15, 18]. Polymorphisms in the SHMT1 gene are associated with increased risk of cardiovascular disease [22, 23] including ischemic stroke [24]. Genetic variation in the SHMT1 gene is further associated with carotid intima-media thickness, a marker for atherosclerosis [25]. However, the impact of SHMT1 on VSMC function and vascular calcification remained illdefined.

Therefore, the present study aimed to elucidate the possible involvement of SHMT1 in osteo-/chondrogenic transdifferentiation and calcification of VSMCs during conditions of hyperphosphatemia in-vitro.

\section{Materials and Methods}

Cell culture of primary human aortic smooth muscle cells

Primary human aortic smooth muscle cells (HAoSMCs) were obtained from Thermo Fisher Scientific [26-28]. Cells were grown to confluence in complete medium containing Waymouth's MB 752/1 medium and Ham's F-12 nutrient mixture (1:1 ratio, Thermo Fisher Scientific), 10\% FBS (Thermo Fisher Scientific), $100 \mathrm{U} / \mathrm{ml}$ penicillin and $100 \mu \mathrm{g} / \mathrm{ml}$ streptomycin (Thermo Fisher Scientific). HAoSMCs were used in all experiments from passages 4 to 11 .

HAoSMCs were transfected with 10 nM SHMT1 siRNA (ID no. s12820, Thermo Fisher Scientific) or with $10 \mathrm{nM}$ negative control siRNA (ID no. 4390843, Thermo Fisher Scientific) using siPORT amine transfection 


\section{Kidney Blood Pressure Research}

agent (Thermo Fisher Scientific) according to the manufacturer's protocol. The cells were used 48 hours (qRT-PCR and antioxidant capacity), 7 days (ALPL activity) or 9 days (calcium deposition) after transfection. Silencing efficiency was determined by quantitative RT-PCR.

HAoSMCs were treated for 24 hours (qRT-PCR) or 7 days (ALPL activity) with $2 \mathrm{mM} \beta$-glycerophosphate (Sigma Aldrich), $10 \mu \mathrm{M}$ TEMPOL (4-hydroxy-TEMPO, stock in DMSO; Sigma-Aldrich) or $10 \mu \mathrm{M}$ TIRON (4, 5-dihydroxy-1, 3-benzenedisulfonic acid disodium salt monohydrate; Sigma-Aldrich). Equal amounts of vehicle were used as control. Treatment with calcification medium $(10 \mathrm{mM} \beta$-glycerophosphate and $1.5 \mathrm{mM} \mathrm{CaCl}$, Sigma-Aldrich) for 9 days was used for the calcium content analysis and Alizarin Red staining [26, 29, 30]. Fresh media with agents were added every 2-3 days.

\section{Quantitative RT-PCR}

Total RNA was isolated from HAoSMCs by using Trizol Reagent (Thermo Fisher Scientific) according to the manufacturer's instructions [31,32]. Reverse transcription of total RNA was performed using oligo(dT) $)_{12-18}$ primers (Thermo Fisher Scientific) and SuperScript III Reverse Transcriptase (Thermo Fisher Scientific). Quantitative RT-PCR was performed with the iCycler iQ ${ }^{\mathrm{TM}}$ Real-Time PCR Detection System (BioRad Laboratories) and $\mathrm{iQ}^{\mathrm{TM}}$ Sybr Green Supermix (Bio-Rad Laboratories) according to the manufacturer's instructions. The following human primers were used (Thermo Fisher Scientific, $5^{\prime} \rightarrow 3^{\prime}$ orientation):

ALPL fw: GGGACTGGTACTCAGACAACG;

ALPL rev: GTAGGCGATGTCCTTACAGCC;

$B A X$ fw: CCCGAGAGGTCTTTTTCCGAG;

$B A X$ rev: CCAGCCCATGATGGTTCTGAT;

$B C L 2$ fw: GGTGGGGTCATGTGTGTGG;

BCL2 rev: CGGTTCAGGTACTCAGTCATCC;

CBFA1 fw: GCCTTCCACTCTCAGTAAGAAGA;

CBFA1 rev: GCCTGGGGTCTGAAAAAGGG;

CYBA fw: CCCAGTGGTACTTTGGTGCC;

CYBA rev: GCGGTCATGTACTTCTGTCCC;

GAPDH fw: GAGTCAACGGATTTGGTCGT;

GAPDH rev: GACAAGCTTCCCGTTCTCAG;

$M M P 2 \mathrm{fw}$ : TACAGGATCATTGGCTACACACC;

MMP2 rev: GGTCACATCGCTCCAGACT;

MSX2 fw: TGCAGAGCGTGCAGAGTTC;

MSX2 rev: GGCAGCATAGGTTTTGCAGC;

NOX4 fw: TGACGTTGCATGTTTCAGGAG;

NOX4 rev: AGCTGGTTCGGTTAAGACTGAT;

SHMT1 fw: TTGCCTCGGAGAATTTCGCC;

SHMT1 rev: GTCCCGCCATAGTATCTCTGG.

The specificity of the PCR products was confirmed by analysis of the melting curves. All PCRs were performed in duplicate and relative mRNA expression was calculated by using the $2^{-\Delta \Delta C t}$ method with GAPDH as housekeeping gene. Results were normalized to the negative control silenced group.

\section{Total antioxidant capacity assay}

Total antioxidant capacity of HAoSMCs was measured in the cell lysate by using the colorimetric antioxidant assay kit (Cayman Chemical) according to the manufacturer's protocols [26,33]. Relative antioxidant capacity was compared to that of Trolox as standard. The results were normalized to total protein concentration as assessed by the Bradford assay (Bio-Rad Laboratories) and to the negative control silenced group.

Alkaline phosphatase (ALPL) activity assay

ALPL activity in HAoSMCs was determined by using the ALP colorimetric assay kit (Abcam) according to the manufacturer's protocol [34]. The results are shown normalized to total protein concentration measured by the Bradford assay (Bio-Rad Laboratories). 


\section{Kidney Blood Pressure Research}

\section{Determination of calcification}

HAoSMCs were decalcified in $0.6 \mathrm{M} \mathrm{HCl}$ for 24 hours at $4^{\circ} \mathrm{C}$. Calcium content in the supernatant was determined by using QuantiChrom Calcium assay kit (BioAssay Systems) according to the manufacturer's protocol. HAoSMCs were lysed with $0.1 \mathrm{M} \mathrm{NaOH} / 0.1 \%$ SDS and protein concentration was measured by the Bradford assay (Bio-Rad Laboratories). Results are shown normalized to total protein concentration $[28,35]$. For Alizarin red staining, HAoSMCs were fixed with 4\% paraformaldehyde/PBS and stained with $2 \%$ Alizarin Red (pH 4.5). The calcified areas are shown as red staining [26].

\section{Statistics}

Data are shown as scatter dot plots and arithmetic means \pm SEM. $\mathrm{N}$ indicates the number of independent experiments performed at different passages of the cells. Normality was tested with Shapiro-Wilk test. Nonnormal datasets were transformed (log, reciprocal or sqrt) prior to statistical testing to provide normality according to Shapiro-Wilk test. Statistical testing was performed by one-way Anova followed by Tukey-test (homoscedastic data) or Games-Howell test (heteroscedastic data). Non-normal data were tested by the SteelDwass method. Two groups were compared by unpaired two-tailed t-test. $\mathrm{P}<0.05$ was considered statistically significant.

\section{Results}

To investigate the role of SHMT1 in vascular calcification, the endogenous expression in primary human aortic smooth muscle cells (HAoSMCs) was suppressed by silencing of the SHMT1 gene followed by additional treatment without and with phosphate. As shown in Fig. 1, SHMT1 mRNA expression was significantly lower in SHMT1 silenced HAoSMCs than in negative control siRNA transfected HAoSMCs. The negative control transfection conditions alone did not significantly affect SHMT1 gene expression in HAoSMCs as compared to untransfected HAoSMCs $(n=5 ; 1.004 \pm 0.042$ a.u. in untransfected HAoSMCs vs. $0.998 \pm 0.059$ a.u. in negative control siRNA silenced HAoSMCs). Phosphate treatment significantly up-regulated SHMT1 mRNA expression in negative control silenced HAoSMCs (Fig. 1).

As illustrated by Alizarin Red staining and quantification of calcium content, treatment with calcification medium increased calcification of negative control silenced HAoSMCs, an effect aggravated in SHMT1 silenced HAoSMCs (Fig. 2A, B). Silencing of SHMT1 alone did not significantly modify calcium deposition in HAoSMCs (Fig. 2A, B). However, SHMT1 knockdown alone was sufficient to significantly increase ALPL activity (Fig. 2C) and mRNA expression of osteogenic transcription factors MSX2 and CBFA1 as well as of osteogenic enzyme $A L P L$ (Fig. 2D-F) in HAoSMCs to similar high levels as phosphate treatment. Furthermore, the phosphate-induced ALPL activity and mRNA expression were significantly augmented following silencing of SHMT1 in HAoSMCs. Silencing of SHMT1 tended to augment MSX2 and CBFA1 mRNA expression in phosphate treated HAoSMCs, an effect, however, not reaching statistical significance. Taken together, silencing of SHMT1 is sufficient to promote osteo-/chondrogenic transdifferentiation and to aggravate the phosphateinduced calcification of VSMCs.

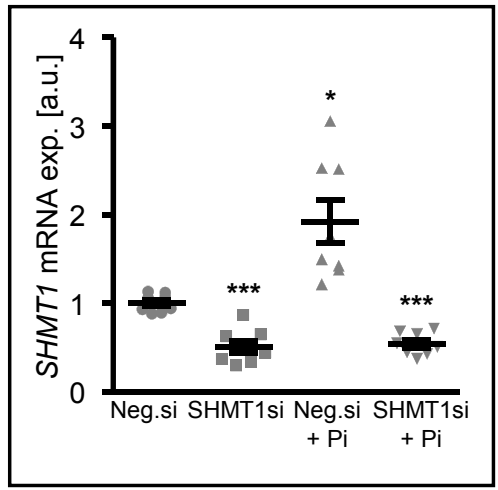

Fig. 1. Phosphate up-regulates cytosolic serine hydroxymethyl transferase 1 (SHMT1) expression in HAoSMCs. Scatter dot plots and arithmetic means \pm SEM $(n=8$; arbitrary units, a.u.) of SHMT1 relative mRNA expression in HAoSMCs following silencing for 48 hours with negative control siRNA (Neg.si) or SHMT1 siRNA (SHMT1si) and additional treatment for 24 hours with control or with $2 \mathrm{mM}$ $\beta$-glycerophosphate (Pi). ${ }^{*}(\mathrm{p}<0.05)$, $* * *(\mathrm{p}<0.001)$ statistically significant vs. negative control silenced HAoSMCs. 


\section{Kidney Blood Pressure Research}

To explore the mechanisms underlying the pro-calcific role of SHMT1 knockdown, another series of experiments analyzed the effects on oxidative stress in HAoSMCs. Total antioxidant capacity (Fig. 3A) was significantly lower and the mRNA expression of oxidative stress markers NOX4 and p22phox encoded by the CYBA gene (Fig. 3B, C) was significantly higher in SHMT1 silenced HAoSMCs than in negative control silenced HAoSMCs. Similarly, silencing of SHMT1 significantly up-regulated matrix metalloproteinase MMP2 mRNA expression (Fig. 3D), a downstream target of oxidative stress in VSMCs. These effects were paralleled by increased $B A X / B C L 2$ mRNA expression ratio as indicator of apoptosis in SHMT1 silenced HAoSMCs (Fig. 3E). Thus, SHMT1 knockdown induces oxidative stress in VSMCs.

Further experiments investigated whether induction of oxidative stress is responsible for the effects of SHMT1 silencing on osteo-/chondrogenic transdifferentiation of VSMCs. As shown in Fig. 4, the increased mRNA expression of osteo-/chondrogenic markers MSX2, CBFA1 and ALPL in SHMT1 silenced HAoSMCs was significantly suppressed following additional treatment with antioxidants TEMPOL or TIRON. Thus, the effects of SHMT1 silencing in promoting osteo-/chondrogenic transdifferentiation of VSMCs are due, at least partially, to induction of cellular oxidative stress.

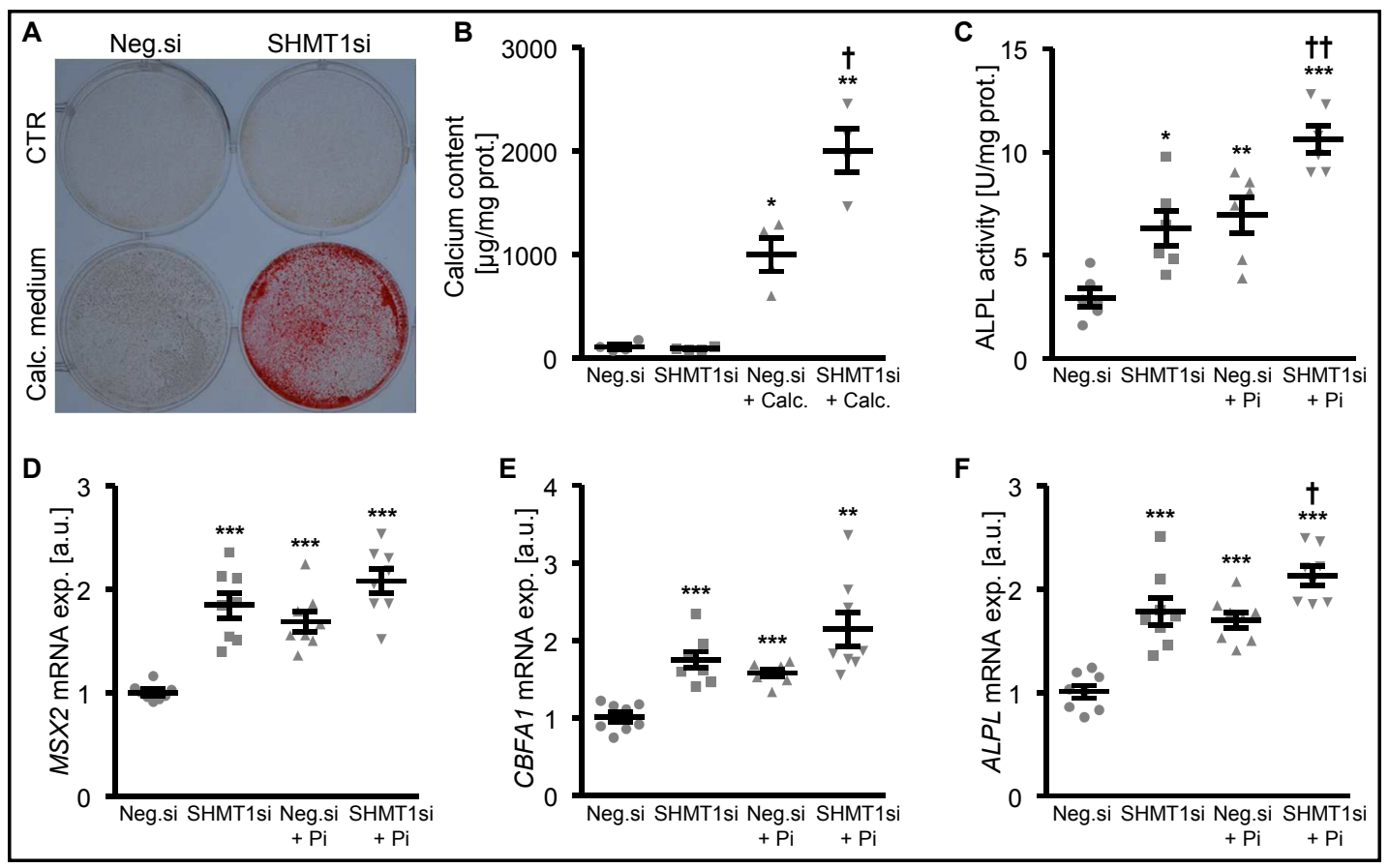

Fig. 2. Silencing of SHMT1 promotes osteoinductive signaling and aggravates phosphate-induced osteo-/ chondrogenic transdifferentiation and calcification of HAoSMCs. A. Representative original images showing Alizarin red staining $(n=3)$ in HAoSMCs following silencing for 9 days with negative control siRNA (Neg. si) or SHMT1 siRNA (SHMT1si) and additional treatment with control or with calcification medium (Calc.). The calcified areas are shown as red staining. B. Scatter dot plots and arithmetic means \pm SEM $(\mathrm{n}=4 ; \mu \mathrm{g} / \mathrm{mg}$ protein) of calcium content in HAoSMCs following silencing for 9 days with negative control siRNA (Neg.si) or SHMT1 siRNA (SHMT1si) and additional treatment with control or with calcification medium (Calc.). C. Scatter dot plots and arithmetic means \pm SEM ( $n=6$; U/mg protein) of ALPL activity in HAoSMCs following silencing for 7 days with negative control siRNA (Neg.si) or SHMT1 siRNA (SHMT1si) and additional treatment with control or with $2 \mathrm{mM} \beta$-glycerophosphate (Pi). D-F. Scatter dot plots and arithmetic means \pm SEM (n=8; arbitrary units, a.u.) of $M S X 2$ (D), CBFA1 (E) and ALPL (F) relative mRNA expression in HAoSMCs following silencing for 48 hours with negative control siRNA (Neg.si) or SHMT1 siRNA (SHMT1si) and additional treatment for 24 hours with control or with $2 \mathrm{mM} \beta$-glycerophosphate (Pi). ${ }^{*}(\mathrm{p}<0.05),{ }^{* *}(\mathrm{p}<0.01),{ }^{* * *}(\mathrm{p}<0.001)$ statistically significant vs. negative control silenced HAoSMCs; $\dagger(\mathrm{p}<0.05), \dagger+(\mathrm{p}<0.01)$ statistically significant vs. negative control silenced and Pi treated HAoSMCs. 


\section{Kidney \\ Blood Pressure Research}

Fig. 3. Silencing of SHMT1 increases oxidative stress and apoptosis in HAoSMCs. A. Scatter dot plots and arithmetic means \pm SEM $(n=6$; arbitrary units, a.u.) of total antioxidant capacity in HAoSMCs following silencing for 48 hours with negative control siRNA (Neg.si) or SHMT1 siRNA (SHMT1si). B-E. Scatter dot plots and arithmetic means \pm SEM (n=6; a.u.) of NOX4 (B), CYBA (C) and MMP2 (D) relative mRNA expression as well as $B A X / B C L 2$ (E) relative mRNA expression ratio in HAoSMCs following silencing for 48 hours with negative control siRNA (Neg.si) or SHMT1 siRNA (SHMT1si). $*(\mathrm{p}<0.05), \quad * *(\mathrm{p}<0.01), \quad * * *(\mathrm{p}<0.001)$ statistically significant vs. negative control silenced HAoSMCs.

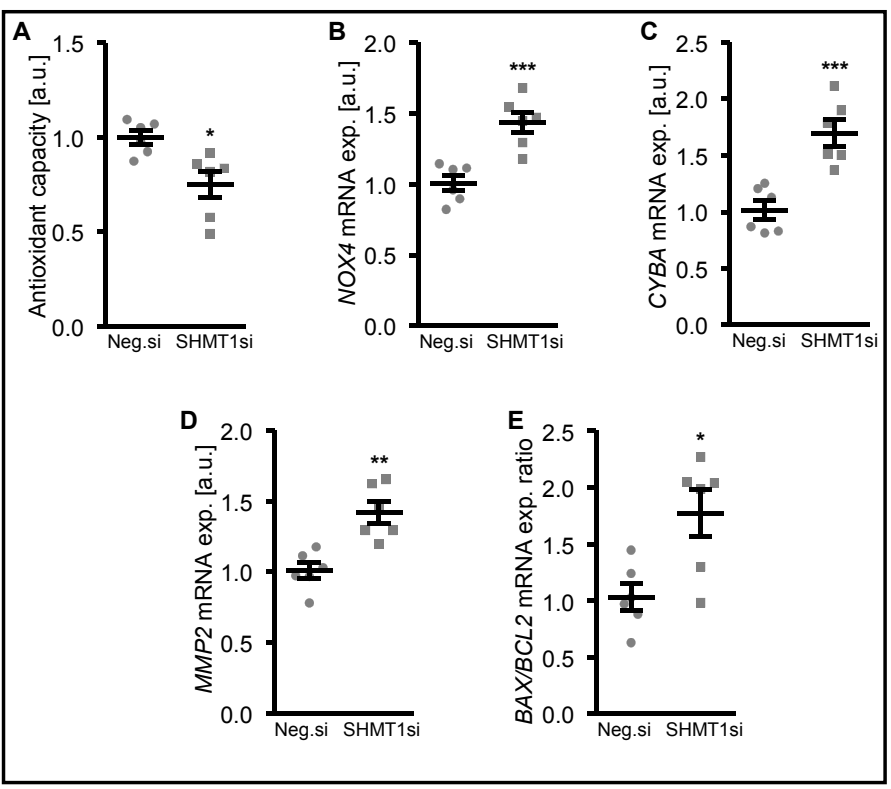

Fig. 4. The osteoinductive effects of SHMT1 silencing in HAoSMCs are blunted in the presence of antioxidants. Scatter dot plots and arithmetic means \pm SEM (n=6; arbitrary units, a.u.) of SHMT1 (A), $M S X 2$ (B), CBFA1 (C) and ALPL (D) relative mRNA expression in HAoSMCs following silencing for 48 hours with negative control siRNA (Neg.si) or SHMT1 siRNA (SHMT1si) and additional treatment with control (CTR), $10 \mu \mathrm{M}$ TEMPOL or $10 \mu \mathrm{M}$ TIRON. ${ }^{*}(\mathrm{p}<0.05),{ }^{* * *}(\mathrm{p}<0.001)$ statistically significant vs. negative control silenced HAoSMCs; $\uparrow(\mathrm{p}<0.05),+\dagger(\mathrm{p}<0.01)$, $\dagger+\dagger(\mathrm{p}<0.001)$ statistically significant vs. control treated and SHMT1 silenced HAoSMCs.

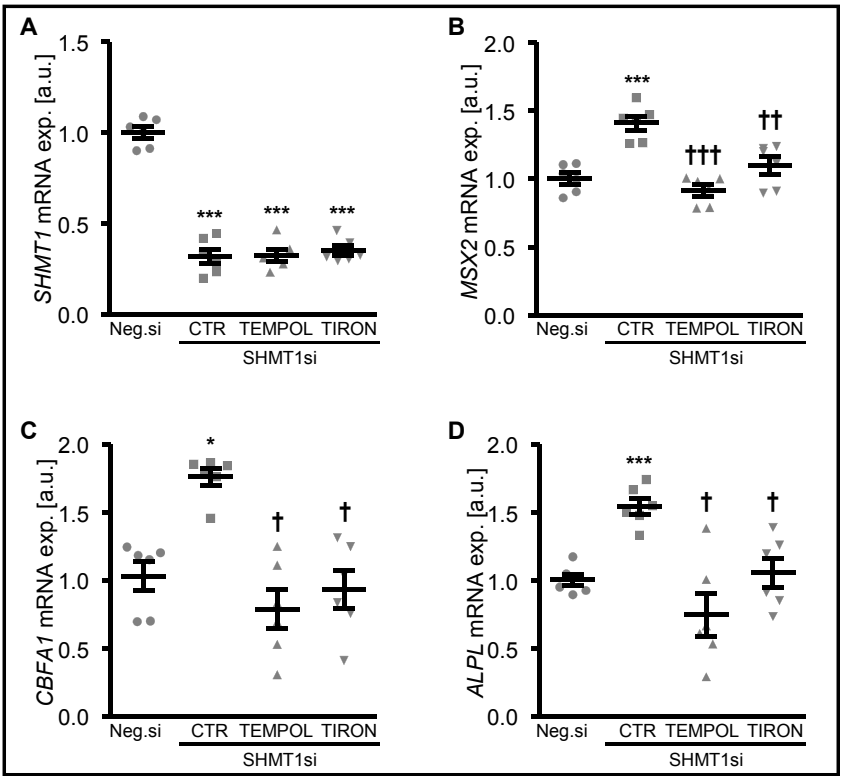

\section{Discussion}

This study reveals a role of SHMT1 in the regulation of VSMC osteo/chondrogenic transdifferentiation and calcification. Phosphate up-regulates SHMT1 expression in VSMCs, while silencing of SHMT1 aggravates phosphate-induced osteo/chondrogenic transdifferentiation and calcification of VSMCs. Silencing of SHMT1 in VSMCs induces oxidative stress and additional treatment with antioxidants blocks the pro-calcific effects of SHMT1 silencing. The present observations, therefore, suggest that vascular SHMT1 is a powerful regulator of the cellular response in pathological conditions such as hyperphosphatemia in CKD [2].

Hyperphosphatemia and dysregulation of mineral homeostasis are critical pathological factors promoting vascular calcification $[2,6]$. In-vitro, elevated extracellular phosphate levels trigger osteo-/chondrogenic transdifferentiation and calcification of VSMCs [36]. We show 


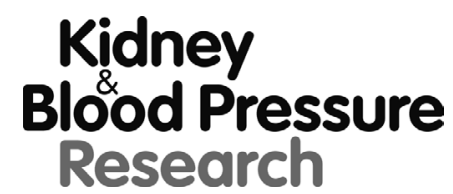
\begin{tabular}{l}
\hline Kidney Blood Press Res 2018;43:1212-1221 \\
\begin{tabular}{l|l}
\hline DOI: 10.1159/000492248 & (c) 2018 The Author(s). Published by S. Karger AG, Basel \\
wublished online: 31 July, 2018 & ww.karger.com/kbr
\end{tabular}
\end{tabular}

Boehme et al.: SHMT1 and Vascular Calcification

here that silencing of SHMT1 augments phosphate-induced osteo-/chondrogenic signaling and calcification of VSMCs. Increased SHMT1 levels in phosphate-treated VSMCs are, thus, apparently necessary to limit the extent of calcification. VSMC calcification models in-vitro require calcium and phosphate supplementation in the cell culture media as substrate for calcification, to permit maximal mineralization [29]. Accordingly, SHMT1 knockdown alone induces osteo-/chondrogenic transdifferentiation, but does not affect mineralization of HAoSMCs, as the substrate for calcification is insufficient. These interpretations are however limited, as the in-vitro findings may not directly reflect vascular calcification in-vivo $[37,38]$. Nonetheless, the present observations indicate that vascular SHMT1 interferes with the osteo-/chondrogenic signaling in phosphate-induced VSMC calcification in-vitro.

Oxidative stress is a critical mediator of vascular calcification [39-41] by inducing osteo-/chondrogenic transdifferentiation of VSMCs [4, 26, 33, 39]. Silencing of SHMT1 in VSMCs induces an imbalance between antioxidant systems and oxidative products, as shown by reduced total antioxidant capacity as well as up-regulation of NOX4 and CYBA mRNA expression, important components of the superoxide-generating NADH/NADPH oxidase system [42].

Oxidative stress may induce osteo-/chondrogenic transdifferentiation of VSMCs via various mechanisms [33, 39, 43-45]. Silencing of SHMT1 is able to increase the expression of downstream effectors of oxidative stress such as matrix gelatinase MMP2 [43], an essential factor in phenotypical transformation of VSMCs and matrix remodeling to initiate mineralization $[44,45]$. Oxidative stress may further promote vascular calcification by inducing apoptosis of VSMCs $[6,46]$. Also, the BAX/BCL2 expression ratio was higher in SHMT1 silenced VSMCs, indicative of increased apoptosis.

Nonetheless, other mechanisms may contribute to the osteoinductive effects of SHMT1 knockdown in VSMCs. The reaction catalyzed by SHMT1 is an important source of glycine $[14,15]$ and hypoglycinemia was shown to contribute to occurrence of vascular calcification and elevated cardiovascular risk in diabetes and CKD [47]. Furthermore, direct targets of SHMT1-mediated methylation [18-21] may be involved in osteoinductive signaling in VSMCs. Conversely, SHMT1 may mediate anti-calcific effects of folate-dependent pathways to protect against vascular calcification [48].

Excessive oxidative stress is prevalent in CKD patients $[49,50]$ and associated with vascular calcification [42] and with increased cardiovascular and all-cause mortality [51]. NADPH oxidase activation is associated with coronary artery calcification [52]. Accordingly, antioxidants may prevent the progression of vascular calcification in CKD [53]. Beyond its role in vascular calcification, SHMT1 may further play an important role in vascular disease in other conditions associated with oxidative stress and apoptosis [49, 50]. Thus, SHMT1 may potentially have a role in systemic changes during disease progression. Further studies are necessary to confirm the potential role of SHMT1 in these conditions.

\section{Conclusion}

SHMT1 knockdown promotes osteo-/chondrogenic transdifferentiation of VSMCs, at least in part, by increasing cellular oxidative stress and oxidative stress-dependent osteoinductive signaling. It aggravates vascular calcification during conditions of hyperphosphatemia in-vitro. The present observations, thus, reveal SHMT1 as novel player in the signaling of vascular calcification.

\section{Acknowledgements}

This work was supported by the European Union Seventh Framework Programme (FP7/2007-2013-603288-SysVasc), the Berlin Institute of Health (BIH) Translational Postdoc 


\section{Kidney \\ Blood Pressure Research}

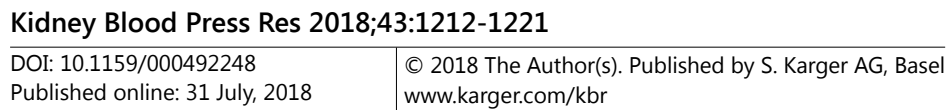

Boehme et al.: SHMT1 and Vascular Calcification

Grant, Deutsche Forschungsgemeinschaft (AL2054/1-1, V02259/2-1), the Open Access

Publication Fund of Charité - Universitätsmedizin Berlin and the Else Kröner-Fresenius-Stiftung. The authors gratefully acknowledge the technical assistance of A. Lange.

\section{Disclosure Statement}

All authors disclose that they have no potential conflict of interest.

\section{References}

1 Shroff R, Long DA, Shanahan C: Mechanistic insights into vascular calcification in CKD. J Am Soc Nephrol 2013;24:179-189.

2 Giachelli CM: The emerging role of phosphate in vascular calcification. Kidney Int 2009;75:890-897.

-3 Rennenberg RJ, Kessels AG, Schurgers LJ, van Engelshoven JM, de Leeuw PW, Kroon AA: Vascular calcifications as a marker of increased cardiovascular risk: a meta-analysis. Vasc Health Risk Manag 2009;5:185-197.

4 Mizobuchi M, Towler D, Slatopolsky E: Vascular calcification: the killer of patients with chronic kidney disease. J Am Soc Nephrol 2009;20:1453-1464.

-5 Tonelli M, Sacks F, Pfeffer M, Gao Z, Curhan G, Cholesterol, Recurrent Events Trial I: Relation between serum phosphate level and cardiovascular event rate in people with coronary disease. Circulation 2005;112:26272633.

-6 Paloian NJ, Giachelli CM: A current understanding of vascular calcification in CKD. Am J Physiol Renal Physiol 2014;307:F891-900.

7 Lang F, Leibrock C, Pelzl L, Gawaz M, Pieske B, Alesutan I, Voelkl J: Therapeutic Interference With Vascular Calcification-Lessons From Klotho-Hypomorphic Mice and Beyond. Front Endocrinol (Lausanne) 2018;9:207.

-8 Lang F, Ritz E, Voelkl J, Alesutan I: Vascular calcification--is aldosterone a culprit? Nephrol Dial Transplant 2013;28:1080-1084.

-9 Voelkl J, Luong TT, Tuffaha R, Musculus K, Auer T, Lian X, Daniel C, Zickler D, Boehme B, Sacherer M, Metzler B, Kuhl D, Gollasch M, Amann K, Muller DN, Pieske B, Lang F, Alesutan I: SGK1 induces vascular smooth muscle cell calcification through NF-kappaB signaling. J Clin Invest 2018; DOI: 10.1172/JCI96477.

10 Lang F, Ritz E, Alesutan I, Voelkl J: Impact of aldosterone on osteoinductive signaling and vascular calcification. Nephron Physiol 2014;128:40-45.

11 Chen NX, Moe SM: Pathophysiology of Vascular Calcification. Curr Osteoporos Rep 2015;13:372-380.

12 Cheng SL, Behrmann A, Shao JS, Ramachandran B, Krchma K, Bello Arredondo Y, Kovacs A, Mead M, Maxson $\mathrm{R}$, Towler DA: Targeted reduction of vascular Msx1 and Msx2 mitigates arteriosclerotic calcification and aortic stiffness in LDLR-deficient mice fed diabetogenic diets. Diabetes 2014;63:4326-4337.

13 Sheen CR, Kuss P, Narisawa S, Yadav MC, Nigro J, Wang W, Chhea TN, Sergienko EA, Kapoor K, Jackson MR, Hoylaerts MF, Pinkerton AB, O’Neill WC, Millan JL: Pathophysiological role of vascular smooth muscle alkaline phosphatase in medial artery calcification. J Bone Miner Res 2015;30:824-836.

14 Girgis S, Nasrallah IM, Suh JR, Oppenheim E, Zanetti KA, Mastri MG, Stover PJ: Molecular cloning, characterization and alternative splicing of the human cytoplasmic serine hydroxymethyltransferase gene. Gene 1998;210:315-324.

-15 Hebbring SJ, Chai Y, Ji Y, Abo RP, Jenkins GD, Fridley B, Zhang J, Eckloff BW, Wieben ED, Weinshilboum RM: Serine hydroxymethyltransferase 1 and 2: gene sequence variation and functional genomic characterization. J Neurochem 2012;120:881-890.

16 Wu XY, Lu L: Vitamin B6 deficiency, genome instability and cancer. Asian Pac J Cancer Prev 2012;13:53335338.

17 MacFarlane AJ, Liu X, Perry CA, Flodby P, Allen RH, Stabler SP, Stover PJ: Cytoplasmic serine hydroxymethyltransferase regulates the metabolic partitioning of methylenetetrahydrofolate but is not essential in mice. J Biol Chem 2008;283:25846-25853. 


\section{Kidney \\ Blood Pressure Research}

Boehme et al.: SHMT1 and Vascular Calcification

18 Kalhan SC: One carbon metabolism in pregnancy: Impact on maternal, fetal and neonatal health. Mol Cell Endocrinol 2016;435:48-60.

19 Zhang L, Meng X, Ju X, Cai H, Li P, Cao Q, Shao P, Qin C, Yin C: One-carbon metabolism pathway gene variants and risk of clear cell renal cell carcinoma in a Chinese population. PLoS One 2013;8:e81129.

20 Wu SS, Lin X, Yuan LQ Liao EY: The Role of Epigenetics in Arterial Calcification. Biomed Res Int 2015;2015:320849.

-21 Paone A, Marani M, Fiascarelli A, Rinaldo S, Giardina G, Contestabile R, Paiardini A, Cutruzzola F: SHMT1 knockdown induces apoptosis in lung cancer cells by causing uracil misincorporation. Cell Death Dis 2014;5:e1525.

22 Wernimont SM, Clark AG, Stover PJ, Wells MT, Litonjua AA, Weiss ST, Gaziano JM, Vokonas PS, Tucker KL, Cassano PA: Folate network genetic variation predicts cardiovascular disease risk in non-Hispanic white males. J Nutr 2012;142:1272-1279.

23 Wernimont SM, Raiszadeh F, Stover PJ, Rimm EB, Hunter DJ, Tang W, Cassano PA: Polymorphisms in serine hydroxymethyltransferase 1 and methylenetetrahydrofolate reductase interact to increase cardiovascular disease risk in humans. J Nutr 2011;141:255-260.

24 Low HQ, Chen CP, Kasiman K, Thalamuthu A, Ng SS, Foo JN, Chang HM, Wong MC, Tai ES, Liu J: A comprehensive association analysis of homocysteine metabolic pathway genes in Singaporean Chinese with ischemic stroke. PLoS One 2011;6:e24757.

25 Sun K, Song J, Liu K, Fang K, Wang L, Wang X, Li J, Tang X, Wu Y, Qin X, Wu T, Gao P, Chen D, Hu Y: Associations between homocysteine metabolism related SNPs and carotid intima-media thickness: a Chinese sib pair study. J Thromb Thrombolysis 2017;43:401-410.

-26 Alesutan I, Feger M, Tuffaha R, Castor T, Musculus K, Buehling SS, Heine CL, Kuro OM, Pieske B, Schmidt K, Tomaschitz A, Maerz W, Pilz S, Meinitzer A, Voelkl J, Lang F: Augmentation of phosphate-induced osteo-/chondrogenic transformation of vascular smooth muscle cells by homoarginine. Cardiovasc Res 2016;110:408-418.

-27 Alesutan I, Musculus K, Castor T, Alzoubi K, Voelkl J, Lang F: Inhibition of Phosphate-Induced Vascular Smooth Muscle Cell Osteo-/Chondrogenic Signaling and Calcification by Bafilomycin A1 and Methylamine. Kidney Blood Press Res 2015;40:490-499.

-28 Alesutan I, Voelkl J, Feger M, Kratschmar DV, Castor T, Mia S, Sacherer M, Viereck R, Borst O, Leibrock C, Gawaz M, Kuro OM, Pilz S, Tomaschitz A, Odermatt A, Pieske B, Wagner CA, Lang F: Involvement Of Vascular Aldosterone Synthase In Phosphate-Induced Osteogenic Transformation Of Vascular Smooth Muscle Cells. Sci Rep 2017;7:2059.

29 Villa-Bellosta R, Millan A, Sorribas V: Role of calcium-phosphate deposition in vascular smooth muscle cell calcification. Am J Physiol Cell Physiol 2011;300:C210-220.

-30 Voelkl J, Tuffaha R, Luong TTD, Zickler D, Masyout J, Feger M, Verheyen N, Blaschke F, Kuro OM, Tomaschitz A, Pilz S, Pasch A, Eckardt KU, Scherberich JE, Lang F, Pieske B, Alesutan I: Zinc Inhibits PhosphateInduced Vascular Calcification through TNFAIP3-Mediated Suppression of NF-kappaB. J Am Soc Nephrol 2018;29:1636-1648.

31 Mia S, Castor T, Musculus K, Voelkl J, Alesutan I, Lang F: Role of AMP-activated protein kinase alpha1 in angiotensin-II-induced renal Tgfss-activated kinase 1 activation. Biochem Biophys Res Commun 2016;476:267-272.

-32 Voelkl J, Alesutan I, Pakladok T, Viereck R, Feger M, Mia S, Schonberger T, Noegel AA, Gawaz M, Lang F: Annexin A7 deficiency potentiates cardiac NFAT activity promoting hypertrophic signaling. Biochem Biophys Res Commun 2014;445:244-249.

-33 Luong TTD, Schelski N, Boehme B, Makridakis M, Vlahou A, Lang F, Pieske B, Alesutan I, Voelkl J: Fibulin-3 Attenuates Phosphate-Induced Vascular Smooth Muscle Cell Calcification by Inhibition of Oxidative Stress. Cell Physiol Biochem 2018;46:1305-1316.

-34 Voelkl J, Alesutan I, Leibrock CB, Quintanilla-Martinez L, Kuhn V, Feger M, Mia S, Ahmed MS, Rosenblatt KP, Kuro OM, Lang F: Spironolactone ameliorates PIT1-dependent vascular osteoinduction in klothohypomorphic mice. J Clin Invest 2013;123:812-822.

35 Alesutan I, Tuffaha R, Auer T, Feger M, Pieske B, Lang F, Voelkl J: Inhibition of osteo/chondrogenic transformation of vascular smooth muscle cells by $\mathrm{MgCl} 2$ via calcium-sensing receptor. J Hypertens 2017;35:523-532. 


\section{Kidney \\ Blood Pressure Research}

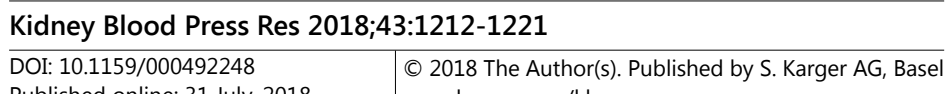

Published online: 31 July, 2018 www.karger.com $/ \mathrm{kbr}$

Boehme et al.: SHMT1 and Vascular Calcification

-36 Giachelli CM: Vascular calcification: in vitro evidence for the role of inorganic phosphate. J Am Soc Nephrol 2003;14:S300-304.

-37 Zebger-Gong H, Muller D, Diercke M, Haffner D, Hocher B, Verberckmoes S, Schmidt S, D'Haese PC, Querfeld $\mathrm{U}:$ 1, 25-Dihydroxyvitamin D3-induced aortic calcifications in experimental uremia: up-regulation of osteoblast markers, calcium-transporting proteins and osterix. J Hypertens 2011;29:339-348.

-38 Haffner D, Hocher B, Muller D, Simon K, Konig K, Richter CM, Eggert B, Schwarz J, Godes M, Nissel R, Querfeld U: Systemic cardiovascular disease in uremic rats induced by 1, 25(OH)2D3. J Hypertens 2005;23:1067-1075.

-39 Byon CH, Javed A, Dai Q, Kappes JC, Clemens TL, Darley-Usmar VM, McDonald JM, Chen Y: Oxidative stress induces vascular calcification through modulation of the osteogenic transcription factor Runx2 by AKT signaling. J Biol Chem 2008;283:15319-15327.

40 Mody N, Parhami F, Sarafian TA, Demer LL: Oxidative stress modulates osteoblastic differentiation of vascular and bone cells. Free Radic Biol Med 2001;31:509-519.

41 Johnson RC, Leopold JA, Loscalzo J: Vascular calcification: pathobiological mechanisms and clinical implications. Circ Res 2006;99:1044-1059.

42 Agharazii M, St-Louis R, Gautier-Bastien A, Ung RV, Mokas S, Lariviere R, Richard DE: Inflammatory cytokines and reactive oxygen species as mediators of chronic kidney disease-related vascular calcification. Am J Hypertens 2015;28:746-755.

43 Valentin F, Bueb JL, Kieffer P, Tschirhart E, Atkinson J: Oxidative stress activates MMP-2 in cultured human coronary smooth muscle cells. Fundam Clin Pharmacol 2005;19:661-667.

44 Chen NX, O’Neill KD, Chen X, Kiattisunthorn K, Gattone VH, Moe SM: Activation of arterial matrix metalloproteinases leads to vascular calcification in chronic kidney disease. Am J Nephrol 2011;34:211219.

-45 Zhao YG, Meng FX, Li BW, Sheng YM, Liu MM, Wang B, Li HW, Xiu RJ: Gelatinases promote calcification of vascular smooth muscle cells by up-regulating bone morphogenetic protein-2. Biochem Biophys Res Commun 2016;470:287-293.

46 Gomez C, Martinez L, Mesa A, Duque JC, Escobar LA, Pham SM, Vazquez-Padron RI: Oxidative stress induces early-onset apoptosis of vascular smooth muscle cells and neointima formation in response to injury. Biosci Rep 2015;35:pii:e00227.

-47 Adeva-Andany M, Souto-Adeva G, Ameneiros-Rodriguez E, Fernandez-Fernandez C, Donapetry-Garcia C, Dominguez-Montero A: Insulin resistance and glycine metabolism in humans. Amino Acids 2018;50:11-27.

48 Nicoll R, Howard JM, Henein MY: A review of the effect of diet on cardiovascular calcification. Int J Mol Sci 2015;16:8861-8883.

49 Poulianiti KP, Kaltsatou A, Mitrou GI, Jamurtas AZ, Koutedakis Y, Maridaki M, Stefanidis I, Sakkas GK, Karatzaferi C: Systemic Redox Imbalance in Chronic Kidney Disease: A Systematic Review. Oxid Med Cell Longev 2016;2016:8598253.

$>50$ Tucker PS, Scanlan AT, Dalbo VJ: Chronic kidney disease influences multiple systems: describing the relationship between oxidative stress, inflammation, kidney damage, and concomitant disease. Oxid Med Cell Longev 2015;2015:806358.

51 Liakopoulos V, Roumeliotis S, Gorny X, Dounousi E, Mertens PR: Oxidative Stress in Hemodialysis Patients: A Review of the Literature. Oxid Med Cell Longev 2017;2017:3081856.

52 Beloqui O, Moreno MU, San Jose G, Pejenaute A, Cortes A, Landecho MF, Diez J, Fortuno A, Zalba G: Increased phagocytic NADPH oxidase activity associates with coronary artery calcification in asymptomatic men. Free Radic Res 2017;51:389-396.

53 Yamada S, Taniguchi M, Tokumoto M, Toyonaga J, Fujisaki K, Suehiro T, Noguchi H, Iida M, Tsuruya K, Kitazono T: The antioxidant tempol ameliorates arterial medial calcification in uremic rats: important role of oxidative stress in the pathogenesis of vascular calcification in chronic kidney disease. J Bone Miner Res 2012;27:474-485. 